\title{
Context-dependent emotional empathy in virtual reality (Empathy in virtual reality)
}

\author{
KPC Kuypers \\ Dept. of Neuropsychology \& Psychopharmacology, \\ Fac. of Psychology \& Neuroscience, \\ Maastricht University, the Netherlands
}

\begin{abstract}
Empathy has been typically studied by means of paradigms including static representations of socially relevant stimuli and it has been acknowledged that there is need for more ecologically valid, dynamic measures. The present study aimed to study implicit ('arousal') and explicit ('concern') emotional empathy in response to positive and negative emotional stories in three virtual reality settings in males and females $(\mathrm{N}=39)$. It was hypothesized that emotional stories would elicit less arousal or implicit emotional empathy in a nature environment compared to a more urbanized environment. Males were hypothesized to score in general lower on empathy measures. Findings showed that emotional empathy was contextdependent and independent of gender. While it has been shown that environmental presence matters when it comes to emotional empathy, the explanation why this specific behavioral pattern was observed still needs to be clarified and suggestions to extend and improve the Virtual Reality Empathy Paradigm have been suggested.
\end{abstract}

Keywords: Emotional empathy, virtual reality, context, gender, immersion

\section{INTRODUCTION}

Empathy is the ability to understand other people's emotions and experience those emotions too. The latter is usually referred to as 'emotional empathy' and the former as 'cognitive empathy' (1-4). Studies have shown that both types have distinct neurobiological underpinnings $(5,6)$. Selective impairment of one of both has been shown in pathologies such as autism spectrum disorder where cognitive empathy is compromised (7) and alcoholism, where emotional empathy is impaired (8).

Typically research has made use of paradigms including static representations of socially relevant stimuli. Prototypical examples of paradigms assessing cognitive empathy are the Reading the Mind in the Eyes test (9) or the Facial Emotional Recognition Test (10) where (parts of) a face expressing either a fully-blown or a morphed emotion are presented. Participants have to choose the word matching the depicted emotion from a predefined number of emotions. In the Multifaceted Empathy test (7) participants are shown pictures of people expressing an emotion and participants have to recognize the emotion ('cognitive empathy') and rate their concern and how this makes them feel ('emotional empathy'). While research using these paradigms -including static stimuli- has shown its merits by for example demonstrating dissociation between cognitive and emotional empathy in adults with Asperger syndrome (7), it has also been argued that there is need for more ecologically valid, dynamic measures (11).

Risko and colleagues (2012) who compared social attention using static and dynamic stimuli showed the added value of using both approaches while stressing not to minimize the value of research using static stimuli either. They showed that while certain attentional processes were 
similar using both kind of stimuli, other, like gaze following was altered when there was a potential for social interaction (12). The latter underscoring the importance of exploring the effects of dynamic stimuli on social behavior.

The Movie for the Assessment of Social Cognition (13) and the Multidimensional Movie Empathy Test (14) are two examples of tasks measuring cognitive and emotional empathy respectively by using film clips in which social interaction is shown. While these stimuli approach daily life situations very closely, the participant is still not part of the social situation. Virtual reality is a means by which this becomes possible, offering promising opportunities to study social behavior (15). Making use of an immersive virtual environment where the environment perceptually surrounds a person offers 'mundane realism', i.e., similarity to situations encountered in everyday life. It is suggested that this increases the engagement within the experimental situation and intensifies the sensitivity to experimental manipulations (15). Immersive virtual environment technology enables the experimental study of social interactions in an accurate and relatively unobtrusive manner (16). Toppenberg et al. (2015) for example studied whether a medical diagnosis and the sexual orientation of a patient (avatar) were related to approaching behavior by the participant in a virtual hospital room. Approach behavior was based amongst other on the interpersonal distance and the speed of approach (17).

When confronted with the emotions of others there can be an automatic and unconscious transfer of emotions between people, known as emotion contagion (18). Exemplary of this is the phenomenon that people feel pain when they see someone in pain or cry when watching a sad movie and see people in distress. Although it has been shown that crying during a movie can paradoxically be experienced as pleasurable (19), being confronted with emotions of others in daily life can be overwhelming and stressful (20). Interestingly, it has been shown that stress recovery is higher when people are in a natural environment and more positive emotional self-reports are provoked in such an environment compared to an urban environment $(21,22)$.

Previous research has shown that emotional empathy can differ between sexes. Women for instance reported to experience the emotions of others with a greater likelihood than men (23). In addition, studies have shown that males consistently score lower on self-reported empathic functioning compared to females (24-27), that women are better at recognizing emotions or 'cognitive empathy' (28), and that brain networks underlying cognitive and emotional empathy show gender differences (29).

The present study was set up to investigate emotional empathy in response to positive and negative emotional stories in virtual reality settings with a different degree of urbanization (park, bar, living room). Based on previous research it was hypothesized that emotional stories would elicit less arousal or implicit emotional empathy in a nature environment (park) compared to a more urbanized environment (bar). There were no specific hypotheses about the effect of environment on explicit emotional empathy ('concern'). Second, it was aimed to test whether there are gender-specific empathic response patterns. Based on previous work it was hypothesized that males would score lower on empathic responses and self-rated empathy compared to females. 


\section{Participants and design}

\section{MATERIALS AND METHODS}

The study was conducted according to a between-subject design with gender (male/female) as between subject factor. In total, 39 healthy volunteers (mean age (SD) 22.4 (2.4); 20 males) were included in the study. The male participants had a mean (SD) age of 22.8 (2.8), the female participants of 22.0 (2.0). Recruitment took place by means of flyers at Maastricht University and through 'Sona Systems', a digital platform (maastricht-fpn.sona-systems.com) for participant recruitment. Exclusion criteria were suffering from mental illness or heart disease.

\section{Procedure}

When interested in the study, individuals were sent the information brochure of the study explaining the aims and procedures. In case of no objection, participants were included. On the test day, upon arrival at the test facilities, participants had the opportunity to ask questions about the study and its procedures. When everything was clear, they signed the informed consent.

The test session started with filling out questionnaires assessing trait empathy by means of the interpersonal reactivity index (IRI) and the empathizing-systemizing questionnaire (EQ/SQ). After this, electrodes to register heart rate were attached to the chest (3-lead ECG, with a leadII configuration) and data was transmitted wirelessly to a laptop. They then were seated in front of a second laptop on which they conducted two empathy tasks, i.e., multifaceted empathy test and the multidimensional movie empathy test (MET). The data of the latter task is reported elsewhere (14). After this, participants did the virtual reality empathy paradigm (VREP) in which both behavioral data and heart rate data were registered. The MET served to validate the VREP.

To compensate for their time investment, participants received either a gift voucher or a participation credit; the latter is part of the Psychology Bachelor training. The study was approved by the Ethical Committee of Psychology of the Faculty of Psychology and Neuroscience of Maastricht University.

\section{Empathy tasks}

\section{Multifaceted Empathy Test}

The Multifaceted Empathy Test (MET) (30) consists of 40 pictures of people conveying a complex emotional state which was positive in $50 \%$ of the pictures and negative in the other half. To assess cognitive empathy, participants had to select, out of 4 words, the emotion word which matched the picture. To assess emotional empathy (EE), participants had to rate on a scale from 1-9 how concerned they were for the person in the picture ('Explicit EE') and how emotionally aroused ('Implicit EE') the picture made them by using the 'Self-Assessment Manikin'. This SAM contains a metaphor for arousal in increasing order, with 5 choice alternatives (31). Dependent variables were the number of correct classified pictures and corresponding reaction times and the Implicit and Explicit EE ratings per valence (7).

\section{Virtual Reality Empathy Paradigm}

By means of Vizard 4.0 enterprise (32 bit) a virtual reality empathy paradigm (VREP) was designed for the current study in order to assess emotional empathy during social interactions in daily life situations. These situations or environments had a different degree of urbanization, as it has been shown previously that a higher degree of urbanization can lead to more stress and nature can lead to faster stress recovery $(21,22)$. The three created environments were: a bar (urbanized environment), a park (nature environment) and a living room (an intermediate environment, i.e., in between urbanized and nature). In these environments 4 avatars were 
positioned. The avatars were 4 realistically looking males $(\mathrm{N}=2)$ and females $(\mathrm{N}=2)$ displaying a positive emotion (joy) or a negative emotion (sadness). The participant saw the three environments through googles and they could enter the environment of choice by saying the number of the environment. The experimenter then gave the participant access to the environment. Once in the environment, 4 avatars were visible and physically approachable. Upon approaching one of the avatars and crossing a certain threshold distance, the avatar started to tell its story, its mouth moved and supporting hand gestures were used. The emotional valence of the story was congruent with the emotion the avatar displayed. When the story ended, after 1 minute on average, a white screen popped up and participants had to answer three subsequent questions. The first one was a control question, asking whether the emotional story they heard was a positive ('happy') story or a negative ('sad') story. Thereafter participants had to answer how concerned they were for the avatar ('explicit emotional empathy') and how much emotional arousal they felt ('implicit emotional empathy'). The latter was assessed by means of self-assessment manikins ('SAM') containing a metaphor for arousal in increasing order, with 5 choice alternatives (31). The former was assessed by means of a 10point scale. After the questions were answered, the participant had to walk to a red dot and $\mathrm{s} /$ he which activated the projection of the 3 environments. The participant had to repeat the whole procedure of picking an environment and an avatar, listening to the story and answering the three questions. In total, 12 stories were presented by 4 avatars in three environments. Heart rate was continuously registered during the task which approximately took approximately 20 minutes. The distance kept from each approached avatar was calculated.

Before starting the task participants were asked to give names (preferably, names of family or friends) to the four avatars in order to increase the (emotional) involvement in the task. These names were shown above the heads of the avatars in the VR-environment. Participants saw the VR environment through a head-mounted display (nVisor ST50, 1280x1024 resolution, $60 \mathrm{~Hz}$ refresh rate) which was connected to a HDMI transmitter/receiver system, worn in a backpack. Participant movements were tracked using a PhaseSpace IMPULSE motion capture system that tracked LED's attached to the head-mounted display. The audio for the VR-task was provided by a single fixed speaker located in the VR-environment.

Dependent variables were the rating of emotional empathy (concern, arousal) and distance kept from the avatar. The categorization of stories by valence was a control measure to test whether participants paid attention to the content of the story.

The 12 stories were rated by independent raters on affective dimensions of valence (emotion intensity of the story on a 10 points-scale) and arousal (arousal experienced on a 5-points SAM scale) when reading the story, not presented in the virtual reality environments. The intensity ratings of the positive stories did not statistically differ between positive stories and had an average $( \pm \mathrm{SE})$ rating of $7.9( \pm 0.2)$; the intensity ratings of the negative stories also did not statistically differ between negative stories and had and average $( \pm \mathrm{SE})$ rating of $6.6( \pm 0.5)$. When comparing positive and negative stories it was shown that the intensity of valence of emotion was rated higher for positive stories compared to negative stories $\left(t_{23}=-2.5=p=\right.$ $0.02)$. The arousal experienced when reading the positive stories statistically differed between stories 3-5 $\left(t_{3}=-5.0=p=0.01\right)$ and stories $5-6\left(t_{3}=3.6=p=0.03\right)$. Arousal ratings for the positives stories did not statistically differ. When averaging the intensity and arousal ratings over stories with the same valence to be presented in the same virtual reality environments, there were no statistically significant differences between ratings, i.e., the stories presented in the three environments had comparable intensity and arousal ratings. 
Heart rate was recorded during the VREP using a wireless Biopac Bionomadix ECG BN-TX device with three electrodes in the lead-II configuration. Data was recorded by means of the Brain Vision recorder 1.20 at a sampling rate of $500 \mathrm{~Hz}$ and preprocessed by means of Vision Analyzer 2.0.

Preprocessing consisted of data filtering (low cut-off: 20; high cut-off: 70, notch: $50 \mathrm{~Hz}$ ) after which R-peaks were automatically detected (threshold: $400 \mu \mathrm{V}$ ). After this process, a visual inspection of the data was conducted to check whether all artifacts were removed. In case of artifacts, these were manually removed. Peaks were exported to Excel where data was averaged per story to a beats per minute value (bpm).

\section{Questionnaires}

\section{Interpersonal Reactivity Index}

The Interpersonal Reactivity Index (IRI) is a 28-item questionnaire consisting of 4 discrete seven-item scales i.e. 'Fantasy', F (tendency to imaginatively transpose oneself into fictional situations), 'Perspective-Taking', PT (tendency to spontaneously adopt the psychological viewpoint of others), 'Empathic Concern', EC (taps the respondents' feelings of warmth, compassion and concern for others), and 'Personal Distress', PD (assesses self-oriented feelings of anxiety and discomfort resulting from tense interpersonal settings). The first two scales are a measure of Cognitive Empathy; the two latter a measure of Emotional Empathy $(32,33)$.

\section{The Empathizing (EQ)-Systemizing Quotient (SQ)}

The EQ-SQ questionnaire consists of 120 statements in a forced choice format (i.e. strongly agree; slightly agree; slightly disagree; strongly disagree). Half of the items form the SQ, the other half the EQ. Both scales contain 20 filler items and 40 'real' items tapering the construct of interest, i.e. respectively 'Systemizing' (= the drive to analyze systems or construct systems) and 'Empathizing' ( = the drive to identify mental states and respond to those with an appropriate emotion). The maximum score on both scales is 80. Previously it has been shown that normal male adults score higher on the SQ and lower on the EQ compared to women (27, $34,35)$.

\section{Statistical analyses}

Data of the VREP (arousal, concern, distance, heart rate), the MET (number correct, arousal, concern), and the empathy questionnaires (IRI and EQ/SQ) were analysed by means of SPSS (version 24.0). The MET and VREP data entered a General Linear Model (GLM) mixed ANOVA with Gender as between subject factor (2 levels) and Valence as within subject factor (2 levels: positive, negative). Environment (3 levels: park, bar, living room) was an additional withinsubject factor in the VREP. In case of main effects of Valence, Environment, or Gender, Bonferroni-corrected pairwise comparisons were conducted; in case of interaction effects subsequent t-tests were conducted to test the origin of the interaction. Empathy-questionnaire data entered two separate multivariate ANOVAs with Gender as fixed factor.

To assess convergent validity of the VREP, Pearson's correlations were calculated between emotional empathy ratings of the VREP (individual stories) and the MET (overall score), separately for negative and positive emotions. In order to test whether implicit and explicit emotional empathy in the MET and VREP were associated, Pearson's correlations were calculated. 
The alpha criterion level of statistical significance for all analyses was set at $p=0.05$; partial $\operatorname{eta}^{2}\left(\eta^{2}\right)$ is reported in case of significant effects to demonstrate the effect's magnitude (0.01: small, 0.06: moderate; 0.14: large).

\section{Multifaceted empathy test Cognitive Empathy}

There was no main effect of Valence $\left(F_{1,37}=3.55, p=0.07, \eta^{2}=0.09\right)$ or Gender $\left(F_{1,37}=1.77, p=\right.$ $\left.0.19, \eta^{2}=0.05\right)$ and no Valence by Gender interaction effect $\left(F_{1,37}=1.11, p=0.30, \eta^{2}=0.03\right)$ on number of correct recognized emotions.

\section{Explicit emotional empathy-Concern}

Analysis revealed a main effect of Valence $\left(F_{1,37}=25.03, p<0.001, \eta^{2}=0.40\right)$ on ratings of concern. Participants felt more concern for individuals displaying a negative emotion compared to a positive emotion. There was no main effect of Gender $\left(F_{1,37}=0.08, p=0.78, \eta^{2}=\right.$ $0.002)$ or an interaction effect between Valence and Gender $\left(F_{1,37}=1.17, p=0.29, \eta^{2}=0.03\right)$ on Concern.

\section{Implicit emotional empathy-Arousal}

Analysis revealed a main effect of Valence $\left(F_{1,37}=21.01, p<0.001, \eta^{2}=0.36\right)$ on arousal ratings. Participants felt more aroused when confronted with negative emotions displayed by individuals displaying compared to a positive emotion. There was no main effect of Gender $\left(F_{1,37}=0.65, p=0.42, \eta^{2}=0.02\right)$ or an interaction effect between Valence and Gender $\left(F_{1,37}=\right.$ $1.34, p=0.25, \eta^{2}=0.03$ ) on Arousal.

\section{Correlation between explicit and implicit emotional empathy}

Pearson's correlations revealed strong statistically significant associations $(p<0.001)$ between explicit and implicit emotional empathy for positive $\left(r_{39}=0.82\right)$ and negative $\left(r_{39}=0.76\right)$ emotions.

\section{Virtual Reality Empathy Paradigm}

Participants correctly (100\%) categorized the stories as positive and negative.

\section{Implicit Emotional Empathy-Arousal}

Analysis showed main effects of Valence $\left(F_{1,37}=60.78, p<0.001, \eta^{2}=0.62\right)$ and Environment $\left(F_{2,74}=5.07, p=0.009, \eta^{2}=0.12\right)$, and a Valence by Environment interaction effect $\left(F_{2,74}=4.95\right.$, $\left.p=0.01, \eta^{2}=0.12\right)$ on Arousal. The valence effect signified that participants were in general more aroused by negative (sad) compared to positive (joyful) stories. The effect of environment indicated that the arousal level was rated higher in the nature environment (park, $p=0.03$ ) and the urbanized environment (bar, $p=0.01$ ) compared to the intermediate environment (living room). Paired sample t-tests to test the origin of the interaction between Valence and Environment demonstrated that arousal ratings were in higher in the urbanized environment when confronted with positive emotions compared to the nature $\left(t_{38}=-2.35 ; p=\right.$ $0.02)$ and intermediate environment $\left(t_{38}=3.49 ; p=0.001\right)$, and that arousal ratings in the nature environment were higher when confronted with negative stories compared to the intermediate environment $\left(t_{38}=2.40 ; p=0.02\right)$ (Figure 1 , panel $\left.\mathrm{A}\right)$.

There was no interaction effect between Valence and Gender $\left(F_{1,37}=0.004, p=0.95, \eta^{2}=0.00\right)$ or Environment and Gender $\left(F_{2,74}=0.28, p=0.76, \eta^{2}=0.007\right)$, and there was also no three-way 
interaction between Valence*Environment*Gender $\left(F_{2,74}=0.18, p=0.83, \eta^{2}=0.005\right)$ on Arousal.

\section{Explicit Emotional Empathy-Concern}

Analysis showed main effects of Valence $\left(F_{1,37}=40.52, p<0.001, \eta^{2}=0.52\right)$ and Environment $\left(F_{2,74}=11.26, p<0.001, \eta^{2}=0.23\right)$ and a Valence by Environment interaction $\left(F_{2,74}=4.65, p=\right.$ $0.01, \eta^{2}=0.11$ ) on Concern. The valence effect showed that in general, negative (sad) stories elicited more concern compared to positive (joyful) stories; the effect of environment signified that the level of concern was higher in the park $(p=0.001)$ and bar $(p<0.001)$ environment compared to the living room. The origin of the interaction effect was tested with paired sample t-tests and it was demonstrated that more concern was felt when confronted with emotional stories in the nature environment compared with the intermediate environment, independent of valence (positive: $t_{38}=2.90 ; p=0.006$; negative: $t_{38}=3.09 ; p=0.004$ ). In addition, concern ratings for positive stories were higher in the urbanized environment compared with the intermediate environment $\left(t_{38}=4.18 ; p<0.001\right)$. Other comparisons did not statistically differ (Figure 1, panel B).

There was no interaction effect between Valence and Gender $\left(F_{1,37}=0.11, p=0.74, \eta^{2}=0.00\right)$ or Environment and Gender $\left(F_{2,74}=0.20, p=0.82, \eta^{2}=0.005\right)$, and there was also no three-way interaction between Valence*Environment*Gender $\left(F_{2,74}=0.08, p=0.92, \eta^{2}=0.002\right)$ on Concern.

\section{Correlation between explicit and implicit emotional empathy}

Pearson's correlations revealed strong statistically significant associations $\left({ }^{*} p<0.001\right)$ between explicit and implicit emotional empathy for the stories heard in the different environments (Table 1).

\begin{tabular}{|l|l|l|l|}
\hline & \multicolumn{3}{|c|}{ Environment } \\
\hline Valence of story & Park & Bar & Living Room \\
\hline+ & $r_{39}=0.79^{*}$ & $r_{39}=0.84^{*}$ & $r_{39}=0.84^{*}$ \\
\hline- & $r_{39}=0.79^{*}$ & $r_{39}=0.81^{*}$ & $r_{39}=0.89^{*}$ \\
\hline
\end{tabular}

Table 1. Pearson's correlations between explicit and implicit emotional empathy in the VREP

\section{Distance}

There were no main or interaction effects of Valence, Environment and Gender on the distance that was kept from the avatar (Figure 1, panel C).

\section{Heart Rate}

Analysis showed a main effect of Valence $\left(F_{1,37}=4.30, p=0.04, \eta^{2}=0.10\right)$ on Heart Rate. In general, positive (joyful) stories caused a higher increase in heart rate compared to negative (sad) stories (Figure 1, panel D).

There was no main effect of Environment $\left(F_{2,74}=1.44, p=0.24, \eta^{2}=0.04\right)$ or Gender $\left(F_{1,37}=\right.$ $\left.0.74, p=0.40, \eta^{2}=0.02\right)$ and no interaction effects between Valence and Gender $\left(F_{1,37}=2.72\right.$, $\left.p=0.11, \eta^{2}=0.07\right)$, Valence and Environment $\left(F_{2,74}=3.05, p=0.053, \eta^{2}=0.08\right)$, or Environment and Gender $\left(F_{2,74}=0.74, p=0.48, \eta^{2}=0.02\right)$, and there was also no three-way interaction between Valence*Environment*Gender $\left(F_{2,74}=2.48, p=0.09, \eta^{2}=0.06\right)$ on Heart Rate. 

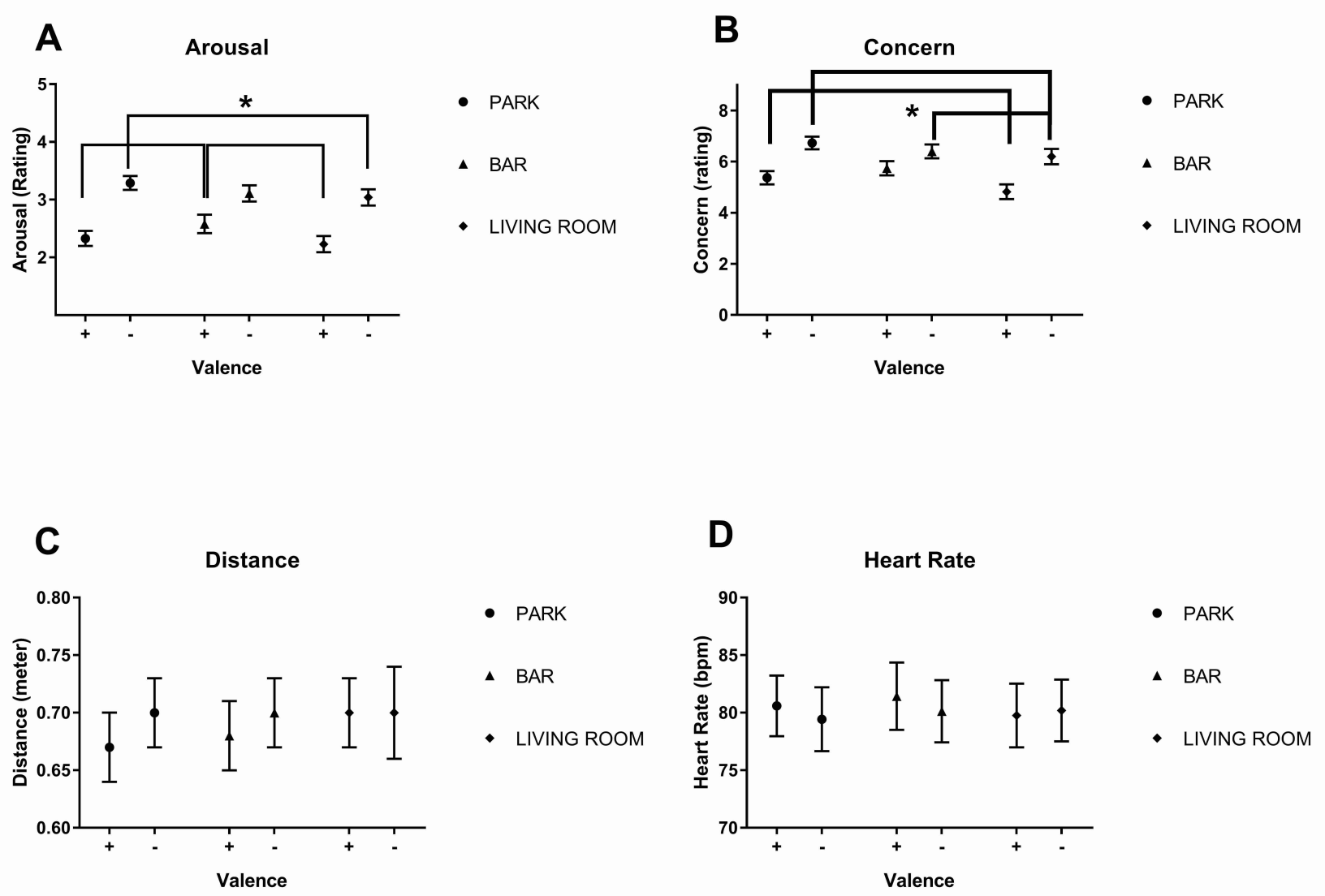

Figure 1. Mean $( \pm S E)$ of arousal (A), concern (B), distance (C) and heart rate (D) during positive $(+)$ and negative $(-)$ emotional stories in three environments in the VREP; ${ }^{*}$ depicts statistically significant $t$-tests at $p=0.05$

\section{Convergent validity}

Pearson's correlations between emotional empathy outcome measures of the MET and VREP demonstrated statistically significant positive correlations between arousal ratings on the MET and VREP and between concern ratings on the MET and VREP (Table 2). 


\begin{tabular}{|c|c|c|c|c|c|c|}
\hline \multicolumn{3}{|l|}{ VREP } & \multicolumn{2}{|c|}{ Arousal VREP-MET } & \multicolumn{2}{|c|}{ Concern VREP-MET } \\
\hline Avatar & Environment & Valence & $r_{39}$ & $P$ & $r_{39}$ & $p$ \\
\hline 1 & \multirow[t]{4}{*}{ Park } & + & 0.48 & 0.002 & 0.56 & $<0.001$ \\
\hline 2 & & & 0.54 & $<0.001$ & 0.51 & 0.001 \\
\hline 3 & & + & 0.51 & 0.001 & 0.59 & $<0.001$ \\
\hline 4 & & - & 0.54 & $<0.001$ & 0.51 & 0.001 \\
\hline 1 & \multirow[t]{4}{*}{ Bar } & + & 0.61 & $<0.001$ & 0.58 & $<0.001$ \\
\hline 2 & & - & 0.58 & $<0.001$ & 0.51 & 0.001 \\
\hline 3 & & + & 0.61 & $<0.001$ & 0.54 & $<0.001$ \\
\hline 4 & & _- & 0.52 & 0.001 & 0.60 & $<0.001$ \\
\hline 1 & \multirow[t]{4}{*}{ LR } & + & 0.58 & $<0.001$ & 0.57 & $<0.001$ \\
\hline 2 & & _ & 0.56 & $<0.001$ & 0.42 & 0.008 \\
\hline 3 & & + & 0.59 & $<0.001$ & 0.67 & $<0.001$ \\
\hline 4 & & 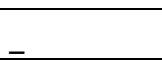 & 0.52 & 0.001 & 0.50 & 0.001 \\
\hline
\end{tabular}

Table 2. Pearson's correlations between outcome measures of the VREP and MET

\section{Interpersonal Reactivity Index}

\section{QUESTIONNAIRES}

Multivariate ANOVA revealed no statistically significant differences between males and females on the two cognitive empathy scales, i.e., Perspective Taking $\left(F_{1,37}=0.003 ; p=0.96\right.$; $\left.\eta^{2}=0.00\right)$ and Fantasy Scale $\left(F_{1,37}=3.81 ; p=0.06 ; \eta^{2}=0.09\right)$. Males and females statistically differed on one of the two emotional empathy scales, i.e., Personal Distress $\left(F_{1,37}=8.82, p=\right.$ $\left.0.005, \eta^{2}=0.19\right)$; it was shown that females scored higher on 'personal distress' compared to males (Figure 2, panel A). There was no statistically significant gender difference on the other emotional empathy scale, i.e., Empathic Concern $\left(F_{1,37}=2.26, p=0.14, \eta^{2}=0.06\right)$.

\section{The Empathizing (EQ)-Systemizing Quotient (SQ)}

Analyses revealed that males scored higher on the systemizing quotient compared to women $\left(F_{1,37}=5.38 p=0.03, \eta^{2}=0.13\right)$. There was no statistically significant difference between males and females on the empathizing quotient $\left(F_{1,37}=0.16, p=0.69, \eta^{2}=0.004\right)$ (Figure 2, Panel B).

\section{A}

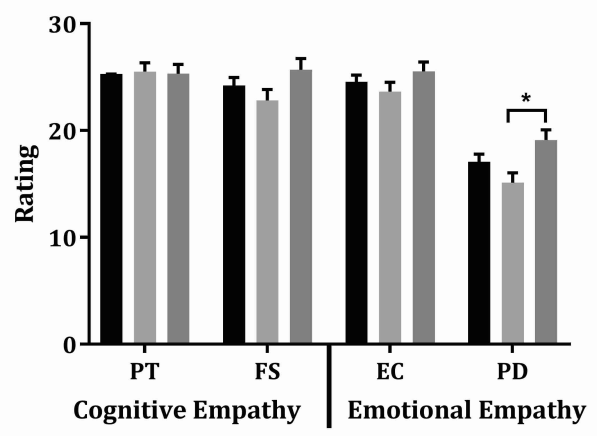

B

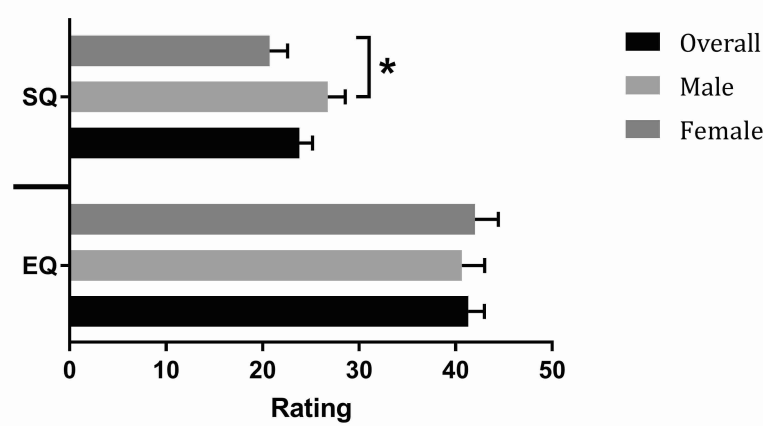

Figure 2. Mean ( \pm SE) of subscales of the Interpersonal Reactivity Index (A) and Empathizing/Systemizing (EQ/SQ) questionnaire for males, females and the total (overall) sample; $\mathrm{PT}=$ Perspective Taking, $\mathrm{FS}=$ Fantasy Scale, $\mathrm{EC}=$ Empathic Concern, $\mathrm{PD}=$ Personal Distress 


\section{DISCUSSION}

The present study aimed to study implicit ('arousal') and explicit ('concern') emotional empathy in response to positive and negative emotional stories in different virtual reality settings in males and females. It was hypothesized that that emotional stories would elicit less arousal (implicit emotional empathy) in a nature environment (park) compared to a more urbanized environment (bar). There were no specific hypotheses about the effect of environment on explicit emotional empathy ('concern'). Males were hypothesized to score in general lower on empathy measures. Findings showed that emotional empathy was contextdependent and independent of gender. Both empathy tests, that is, the MET and the newly designed VREP, measured the same construct as shown by high convergent validity.

\section{CONTEXT-DEPENDENT EMOTIONAL EMPATHY}

In the urbanized (bar) environment, implicit emotional empathy, i.e. the arousal felt when confronted with positive emotional stories, was rated higher compared to when stories were heard in the nature and the intermediate environment. This was in line with the hypothesis, based on previous research, demonstrating a faster recovery from stress (arousal) in nature environments $(21,22)$. However, when confronted with negative emotional stories, more arousal was reported in nature compared to the intermediate environment. This finding cannot be explained by stress-reduction by a nature environment. It was assumed in the present study that reported arousal levels would translate to feelings of distress, however this is not certain.

Physiological responses could provide more information about this distress-arousal association. The current study showed that heart rate decelerated in response to negative stories. This is in line with previous findings showing larger heart rate decelerations in response to negative affect stimuli (36) which could be attributed to an increased allocation of attention when presented with high arousing stimuli (37). The effect in the present study was however context-independent, in contrast to the behavioral data. It has been shown that another physiological measure, i.e., skin conductance might be more sensitive to the behavioral changes in emotional paradigms since this measure has been shown to increase with reported affective arousal $(10,38)$. It is known that the skin conductance response is associated with the stress response (39) and therefore adding this physiological measure to the VREP would contribute in understanding the context-dependent nature of the implicit emotional empathy response.

Explicit emotional empathy was shown to be higher, independent of valence, in the nature environment compared to the intermediate environment, and to be higher in response to positive stories heard in the urbanized environment compared to the intermediate environment. Although this pattern is slightly different from the pattern observed for arousal, both subtypes of emotional empathy seemed to correlate strongly. In other words, the feeling they experienced, elicited by the story, was as intense as the concern they felt for the person telling the story.

\section{TRAIT AND STATE EMOTIONAL EMPATHY AND GENDER}

Empathy trait questionnaires (IRI, EQ/SQ) revealed in general no differences between gender except from higher ratings by females on 'Personal Distress', a subscale of the IRI, and higher ratings for males compared to females on Systemizing, a subscale of the EQ/SQ. The latter finding was party in line with research from others showing males to score higher on the systemizing $(27,35)$; the present study failed, in contrast to the previously mentioned research, to show differences between sexes on empathizing. Although not statistically tested, the females in the present study seemed to score on average lower compared to the means 
found in other studies (35). With respect to the IRI, a similar response pattern has been shown in the past, in a sample of male and female nurses (40). It could also be argued that the absence of a difference between sexes in self-reported empathy traits in both the IRI and EQ/SQ was sample-specific and due to the fact that it were mostly psychology students who might be more alike in terms of personality. Although this is highly speculative and remains to be tested, support for this suggestion can be found in a previous study showing that certain personality traits were associated with the specialization nurses chose during their training (41). With respect to the absence of gender differences in behavioral measures of empathy, there is a void of research, so that a reference to compare with is largely missing. While females have reported a higher likelihood of feeling the feelings of others (23) more work is needed in this field.

\section{LIMITATIONS AND FUTURE STUDIES}

While the strength of the present study is that emotional empathy in response to dynamic 'multisensory' stimuli in a number of immersive virtual environments was studied, the range of emotions was limited to sadness and happiness. Extending the current paradigm with the basic emotions 'anger', 'fear', 'surprise', and 'disgust' (42) and taking into account the recent findings on 4 culturally common facial expression patterns (43) will allow us to study cross-cultural a broad spectrum of emotions, in an ecologically valid environment.

The addition of questions about the experience of physical and spatial presence in virtual reality to the VREP could also provide valuable information about gender-specific reactions in VR paradigms since it has previously been shown that men experienced more physical presence, involvement ('cognitive engagement') and a higher sense of being there than women in a VR situation (39). Interestingly one study also showed that a subscale of the interpersonal reactivity index, 'Fantasy', mediated gender differences in physical presence, though not social presence (44). Although we did not show gender differences in the VREP nor on the Fantasy scale, it has to be explored whether this holds with other samples and a broader scale of emotions.

In the present study, heart rate was included as a physiological measure of emotional empathy. Research has shown that while heart rate is sensitive to the valence of a stimulus, the skin conductance response is more sensitive to arousal $(38,45,46)$. In addition, these physiological data could be used to provide feedback to the participant about the response given to certain emotions $(45,47)$ and thereby improving empathy, since it has been shown that empathy is learnable (48). A therapeutic application of the VREP could be improving emotional empathy in people with alcoholism since this ability has been shown to be disturbed (8). Furthermore, using this paradigm in imaging research with the given population could lead to a better understanding about the underlying neurobiology of their disturbed emotional empathy.

\section{CONCLUSIONS}

In conclusion, it has been shown that environmental presence matters when it comes to emotional empathy, since implicit and explicit emotional empathy differed between virtual environments that had a different degree of urbanization. The explanation why this specific behavioral pattern was observed still needs to be clarified and suggestions to extend and improve the VREP have been suggested.

\section{ACKNOWLEDGEMENTS}

The author thanks the technical department of the Faculty of Psychology and Neuroscience of Maastricht University, in particular J. Ronner and R. Benning, for programming the virtual 
reality paradigm, S. Hazenberg and S. Geelkens for collecting the data, the independent raters of the stories, and the 4 people $(\mathrm{CvH}, \mathrm{PH}, \mathrm{JR}, \mathrm{JW})$ who lend their voice to the avatars.

\section{COMPETING INTERESTS STATEMENT}

None.

\section{FUNDING}

This research did not receive any specific grant from funding agencies in the public, commercial, or not-for-profit sectors.

\section{References}

Decety J, Jackson PL. A social-neuroscience perspective on empathy. Current Directions in Pyschological Science. 2006;15(2):54-8.

Decety J. Dissecting the neural mechanisms mediating empathy. Emotion review. 2011;3(1):92-108.

Gyurak A, Gross JJ, Etkin A. Explicit and implicit emotion regulation: A dual-process framework. Cognition and emotion. 2011;25(3):400-12.

Shamay-Tsoory SG. The Neural Bases for Empathy. The Neuroscientist. 2011 February 1, 2011;17(1):18-24.

Shamay-Tsoory SG, Aharon-Peretz J, Perry D. Two systems for empathy: a double dissociation between emotional and cognitive empathy in inferior frontal gyrus versus ventromedial prefrontal lesions. Brain. 2009;132:617-27.

Cox CL, Uddin LQ, Di Martino A, Castellanos FX, Milham MP, Kelly C. The balance between feeling and knowing: affective and cognitive empathy are reflected in the brain's intrinsic functional dynamics. Social Cognitive and Affective Neuroscience. 2012;7(6):727-37.

Dziobek I, Rogers K, Fleck S, Bahnemann M, Heekeren HR, Wolf OT, et al. Dissociation of cognitive and emotional empathy in adults with Asperger syndrome using in the multifaceted empathy test. Journal of autism and developmental disorders. 2008;38:464-73.

Maurage P, Grynberg D, Noël X, Joassin F, Philippot P, Hanak C, et al. Dissociation Between Affective and Cognitive Empathy in Alcoholism: A Specific Deficit for the Emotional Dimension. Alcoholism: Clinical and Experimental Research. 2011;35(9):1662-8.

Baron-Cohen S, Wheelwright S, Hill J, Raste Y, Plumb I. The 'reading the mind in the eyes' test revised version: a study with normal adults, and adults with Asperger syndrome or high-functioning autism. Journal of child psychology and psychiatry, and allied disciplines. 2001;42(2):241-51.

Lang PJ, Greenwald MK, Bradley MM, Hamm AO. Looking at pictures: Affective, facial, visceral, and behavioral reactions. Psychophysiology. 1993;30(3):261-73.

Dziobek I. Comment: Towards a More Ecologically Valid Assessment of Empathy. Emotion Review. 2012 2012/01/01;4(1):18-9.

Risko EF, Laidlaw KEW, Freeth M, Foulsham T, Kingstone A. Social attention with real versus reel stimuli: toward an empirical approach to concerns about ecological validity. Frontiers in Human Neuroscience. 2012 05/25

$01 / 10 /$ received

05/07/accepted;6:143. PubMed PMID: PMC3360477.

Dziobek I, Fleck S, Kalbe E, Rogers K, Hassenstab J, Brand M, et al. Introducing MASC: a movie for the assessment of social cognition. J Autism Dev Disord. 2006 Jul;36(5):623-36. PubMed PMID: 16755332. Epub 2006/06/07. eng.

Kuypers KPC. Emotional empathic responses to dynamic negative affective stimuli is gender-dependent. Frontiers in Psychology, Emotion Science. 2017;doi: 10.3389/fpsyg.2017.01491.

Blascovich J, Loomis J, Beall AC, Swinth KR, Hoyt CL, Bailenson JN. Immersive virtual environment technology as a methodologial tool for social psychology. Psychological Inquiry: An international journal for the advancement of psychological theory. 2002;13(2):103-24.

Gillath O, McCall C, Shaver PR, Blascovich J. What can virtual reality teach us about prosocial tendencies in real and virtual environments? Media Psychology. 2008;11(2):259-82. 
Toppenberg HL, Bos AER, Ruiter RAC, Wigboldus DHJ, Pryor JB. HIV-related stigma in social interactions: Approach and avoidance behaviour in a virtual environment. European Journal of Social Psychology. 2015;45(2):169-79.

Hatfield E, Cacioppo JT, Rapson RL. Emotional Contagion. Current Directions in Psychological Science. 1993;2(3):96-100.

Hanich J, Wagner V, Shah M, Jacobsen T, Menninghaus W. Why we like to watch sad films. The pleasure of being moved in aesthetic experiences. Psychology of Aesthetics, Creativity, and the Arts. 2014;8(2):130.

Schieman S, Turner HA. "When Feeling Other People's Pain Hurts": The Influence of Psychosocial Resources on the Association between Self-Reported Empathy and Depressive Symptoms. Social Psychology Quarterly. 2001;64(4):376-89.

Ulrich RS, Simons RF, Losito BD, Fiorito E, Miles MA, Zelson M. Stress recovery during exposure to natural and urban environments. Journal of Environmental Psychology. 1991 1991/09/01/;11(3):201-30.

Hartig T, BÖÖK A, Garvill J, Olsson T, GÄRling T. Environmental influences on psychological restoration. Scandinavian Journal of Psychology. 1996;37(4):378-93.

Brody LR, Hall J. Gender and emotion in context. In: Lewis M, Haviland-Jones JM, Barrett LF, editors. Handbook of Emotion. New York: The Guidlford Press; 2008. p. 395-408.

Reniers RLEP, Corcoran R, Drake R, Shryane NM, Völlm BA. The QCAE: A Questionnaire of Cognitive and Affective Empathy. Journal of Personality Assessment. 2011 2011/01/01;93(1):84-95.

Mestre MV, Samper P, Frías MD, Tur AM. Are women more empathic than men? A longitudinal study in adolescence. The Spanish journal of psychology. 2009;12(1):76-83.

Wright DB, Skagerberg EM. Measuring Empathizing and Systemizing with a Large US Sample. PLoS One. 2012;7(2).

Baron-Cohen S, Richler J, Bisarya D, Gurunathan N, Wheelwright S. The systemizing quotient: an investigation of adults with Asperger syndrome or high-functioning autism, and normal sex differences. Philosophical transactions of the Royal Society of London Series B Biological sciences. 2003;358:361-74.

Kret ME, De Gelder B. A review on sex differences in processing emotional signals. Neuropsychologia. 2012 6//;50(7):1211-21.

Christov-Moore L, Simpson EA, Coudé G, Grigaityte K, Iacoboni M, Ferrari PF. Empathy: Gender effects in brain and behavior. Neuroscience \& Biobehavioral Reviews. 2014 10//;46, Part 4:604-27.

Hurlemann R, Patin A, Onur OA, Cohen MX, Baumgartner T, Metzler S, et al. Oxytocin enhances amygdaladependent, socially reinforced learning and emotional empathy in humans. The journal of neuroscience. 2010;30(14):4999-5007.

Bradley MM, Lang PJ. Measuring emotion: the self-assessment manikin and the semantic differential. Journal of Behavioral Therapy and Experimental Psychiatry. 1994;25(1):49-59.

Davis MH. Measuring individual differences in empathy: evidence for a multidimensional approach. Journal of personalilty and social psychology 1983;44:113-26.

De Corte K, Buysse A, Verhofstad LL, Roeyers H, Ponnet K, Davis MH. Measuring empathic tendencies: Reliability and validity of the Dutch version of the interpersonal reactivity index. Psychologica Belgica. 2007;47:235-60.

Claridge G, McDonald A. An investigation into the relationships between convergent and divergent thinking, schizotypy, and autistic traits. Personality and Individual Differences. 2009;46:794-9.

Groen Y, Fuermaier ABM, Den Heijer AE, Tucha O, Althaus M. The Empathy and Systemizing Quotient: The Psychometric Properties of the Dutch Version and a Review of the Cross-Cultural Stability. Journal of Autism and Developmental Disorders. 2015;45(9):2848-64.

Anttonen J, Surakka V. Emotions and heart rate while sitting on a chair. Proceedings of the SIGCHI Conference on Human Factors in Computing Systems; Portland, Oregon, USA. 1055040: ACM; 2005. p. 491-9.

Detenber BH, Simons RF, Bennett GG. Roll 'em!: The effects of picture motion on emotional responses. Journal of Broadcasting \& Electronic Media. 1998 1998/01/01;42(1):113-27.

Greenwald MK, Cook EW, Lang PJ. Affective judgement and psychophysiological response: dimensional covariation in the evaluation of pictorial stimuli. Journal of Psychophysiology. 1989 //;3:51-64. 
Montero-López E, Santos-Ruiz A, García-Ríos MC, Rodríguez-Blázquez R, Pérez-García M, Peralta-Ramírez MI. A virtual reality approach to the Trier Social Stress Test: Contrasting two distinct protocols. Behavior Research Methods. 2016 March 01;48(1):223-32.

Becker H, Sands D. The relationship of empathy to clinical experience among male and female nursing students. The Journal of nursing education. 1988 May;27(5):198-203. PubMed PMID: 2839638. Epub 1988/05/01. eng.

Kennedy B, Curtis K, Waters D. Is there a relationship between personality and choice of nursing specialty: an integrative literature review. BMC Nursing. 2014 November 28;13(1):40.

Ekman P, Levenson RW, Friesen WV. Autonomic nervous system activity distinguishes among emotions. Science (New York, NY). 1983 Sep 16;221(4616):1208-10. PubMed PMID: 6612338. Epub 1983/09/16. eng.

Jack RE, Sun W, Delis I, Garrod OG, Schyns PG. Four not six: Revealing culturally common facial expressions of emotion. Journal of experimental psychology General. 2016 Jun;145(6):708-30. PubMed PMID: 27077757. Epub 2016/04/15. eng.

Felnhofer A, Kothgassner OD, Hauk N, Beutl L, Hlavacs H, Kryspin-Exner I. Physical and social presence in collaborative virtual environments: Exploring age and gender differences with respect to empathy. Computers in Human Behavior. 2014 2014/02/01/;31:272-9.

Prendinger $\mathrm{H}$, Ishizuka M. The empathic companion: A character-based interface that addresses users'affective states. Applied Artificial Intelligence. 2005;19(3-4):267-85.

Mauss IB, Robinson MD. Measures of emotion: A review. Cognition \& emotion. 2009;23(2):209-37. PubMed PMID: PMC2756702.

Fukushima H, Terasawa Y, Umeda S. Association between interoception and empathy: Evidence from heartbeatevoked brain potential. International Journal of Psychophysiology. 2011 2011/02/01/;79(2):259-65.

Georgi E, Petermann F, Schipper M. Are empathic abilities learnable? Implications for social neuroscientific research from psychometric assessments. Social Neuroscience. 2014 2014/01/02;9(1):74-81. 